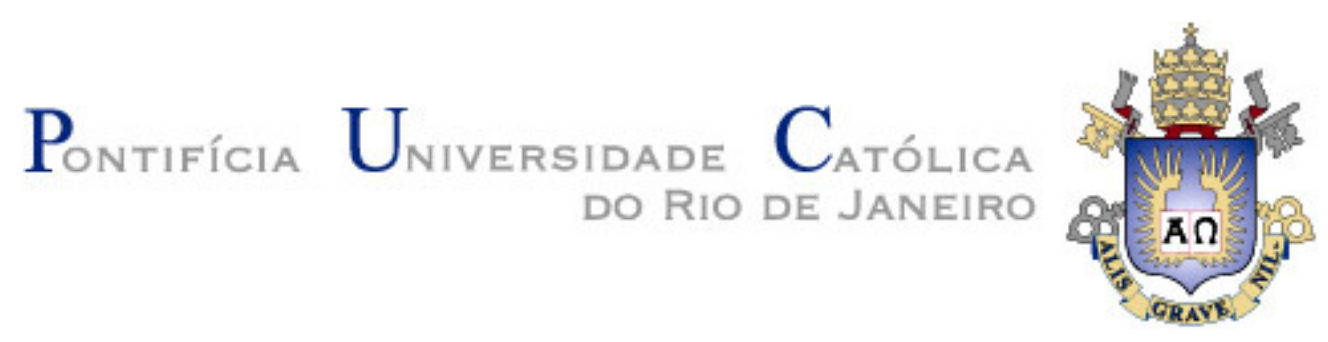

Martin Alexander Purizaga Sandoval

\title{
Análise determinística e probabilística da estabilidade de taludes
}

\section{Dissertação de Mestrado}

Dissertação apresentada como requisito parcial para obtenção do título de Mestre pelo Programa de Pós-Graduação em Engenharia Civil da PUC-Rio.

Orientador: Prof. Celso Romanel 


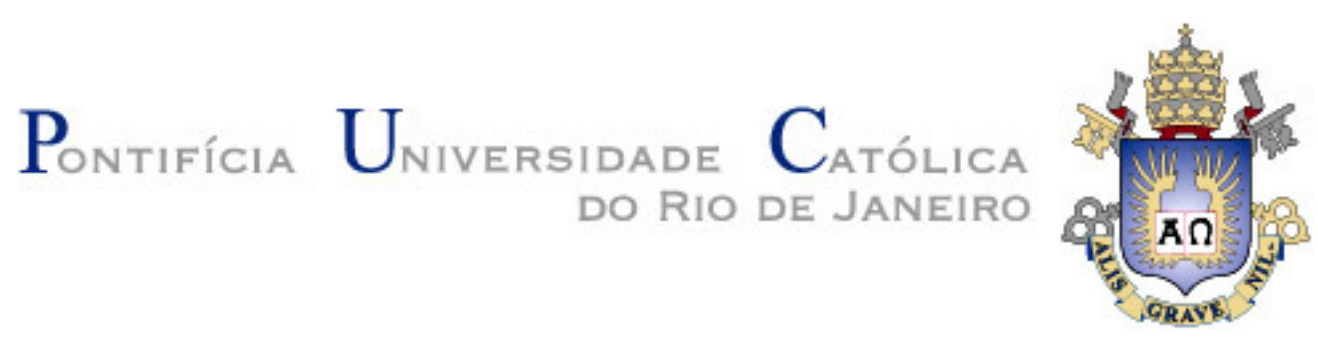

Martin Alexander Purizaga Sandoval

\author{
Análise determinística e probabilística \\ da estabilidade de taludes
}

Dissertação apresentada como requisito parcial para obtenção do título de Mestre pelo Programa de PósGraduação em Engenharia Civil da PUC-Rio. Aprovada pela Comissão Examinadora abaixo assinada.

Prof. Celso Romanel Orientador

Departamento de Engenharia Civil - PUC-Rio

Profa. Michéle Dal Toé Casagrande Departamento de Engenharia Civil - PUC-Rio

Profa. Bernadete Ragoni Danziger UERJ

Prof. José Eugênio Leal Coordenador Setorial do Centro Técnico

Científico - PUC-Rio

Rio de Janeiro, 13 de Julho de 2012 
Todos os direitos reservados. É proibida a reprodução total ou parcial do trabalho sem autorização da universidade, do autor e do orientador.

\section{Martin Alexander Purizaga Sandoval}

Graduou-se em Engenharia Civil na Universidade Ricardo Palma (Lima-Peru) em 2006. Trabalhou na área de projetos e supervisão de obras de mineração. Ingressou em 2010 no curso de mestrado em Engenharia Civil da Pontifícia Universidade Católica de Rio de Janeiro, na área de Geotecnia.

Ficha Catalográfica

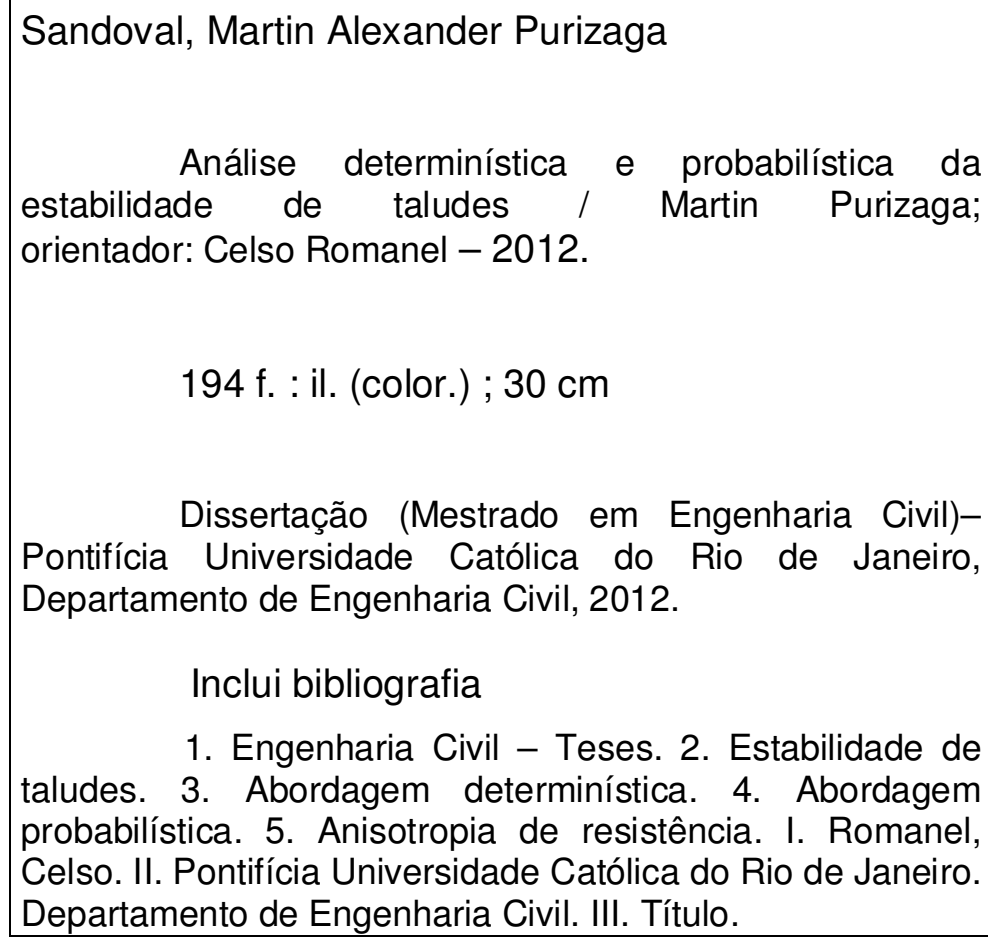

1. Engenharia Civil - Teses. 2. Estabilidade de taludes. 3. Abordagem determinística. 4. Abordagem probabilística. 5. Anisotropia de resistência. I. Romanel, Celso. II. Pontifícia Universidade Católica do Rio de Janeiro. Departamento de Engenharia Civil. III. Título. 


\section{Agradecimentos}

Agradeço a Deus por sempre me acompanhar em todo momento da minha vida.

Agradeço a minha maravilhosa família, que sempre estiveram para dar-me ânimos e força quando eu mais precisei, além do amor brindado durante todo este tempo longe de casa.

Ao Professor Celso Romanel pela orientação, companheirismo e exemplo de profissional.

Aos representantes da banca examinadora formada pelas professoras; Michéle Dal Toé Casagrande e Bernadete Ragoni Danziger, cujas observações ajudarão no meu crescimento profissional.

Aos meus colegas do curso de Mestrado em Engenharia Civil da PUC-Rio, em especial a Jackeline Castañeda; Lidiane Alves; Andrea Rojas; Alexandre Brandão e Ricardo Amado, por fazer este tempo mais simples para mim, e principalmente pela valiosa amizade.

Aos amigos que desde o Peru sempre me apoiaram em todo este tempo, como a Sra. Rosa Chung e Fiorella Rangel, fico grato com vocês.

A Rita de Cassia e Paula Enoy pelas informações fornecidas antes e durante o Mestrado.

À PUC-Rio e a CAPES pela ajuda financeira indispensável para o desenvolvimento deste trabalho. 


\section{Resumo}

Sandoval, Martin Alexander Purizaga; Romanel, Celso (Orientador) Análise determinística e probabilística da estabilidade de taludes. Rio de Janeiro, 2012. 194p. Dissertação de Mestrado - Departamento de Engenharia Civil, Pontifícia Universidade Católica do Rio de Janeiro.

$\mathrm{Na}$ abordagem determinística compara-se o método do equilíbrio limite com o método dos elementos finitos na análise 2D da estabilidade de taludes em solo, determinando-se o valor do fator de segurança para vários exemplos de aplicação. No método dos elementos finitos quantifica-se a região da potencial superfície de ruptura que se comporta sob extensão, analisando-se a ocorrência de anisotropia de resistência ao longo da potencial superfície de deslizamento para situações envolvendo diferentes tipos de solo, ângulos de inclinação do talude e sequências de escavação. Conclui-se que o efeito da anisotropia é pouco significativo quando o ângulo de atrito do solo exceder a $10^{0}$. Por outro lado, devido à crescente aplicação da abordagem probabilística na prática da engenharia geotécnica, foram realizadas análises de confiabilidade de taludes empregando os métodos de Monte Carlo, do hipercubo latino e o método das estimativas pontuais alternativas, comparando-se os diferentes resultados obtidos e discutindo as principais vantagens da aplicação destes métodos.

\section{Palavras-chave}

Estabilidade de taludes; abordagem determinística; abordagem probabilística; anisotropia de resistência. 


\section{Abstract}

Sandoval, Martin Alexander Purizaga; Romanel, Celso (Advisor) Deterministic and probabilistic analysis of slope stability, Rio de Janeiro, 2012. 194p. MSc. Dissertation - Departamento de Engenharia Civil. Pontifícia Universidade Católica do Rio de Janeiro.

In the deterministic approach the limit equilibrium method and the finite element method are compared for 2D analysis of soil slope stability, calculating the value of the safety factor in several numerical examples. In the finite element method the region of soil under strain extension is determined and the occurrence of strength anisotropy is investigated along the potential sliding surface for situations involving different types of soil, angles of slope inclination and excavation sequences. It is concluded that the effect of the anisotropy is negligible

when the angle of friction is higher than $10^{\circ}$. Moreover, due to the increasing application of the probabilistic approach in the practice of geotechnical engineering, some reliability analyses were carried out using the Monte Carlo method, the Latin hypercube method and the method of alternative point estimates, comparing their different outputs and discussing the main advantages of their application in the analysis of soil slope stability problems.

\section{Keywords}

Slope stability; deterministic approach; probabilistic approach; strength anisotropy. 


\section{Sumário}

1 Introdução 22

1.1. Objetivos 24

1.2. Organização da dissertação 25

2 Revisão Bibliográfica 26

2.1. Métodos de análise de estabilidade de taludes 26

2.1.1. Métodos de equilíbrio limite 26

2.1.2. Métodos dos elementos finitos 36

2.1.3. Métodos da análise limite 41

2.2. Influência da anisotropia na análise de estabilidade de taludes 43

2.3. Métodos probabilísticos para avaliação da estabilidade de taludes 45

2.3.1. Métodos de análise probabilística 47

3 Abordagem determinística na análise de estabilidade de taludes 49

3.1. Método dos elementos finitos na estabilidade de talude 49

3.1.1. Método Direto 51

3.1.2. Método do Equilíbrio Limite Aperfeiçoado 54

3.1.3. Justificativa para o uso do MEF 54

3.1.4. Requisitos para a análise dos elementos finitos 55

3.1.5. Mecanismos de ruptura $\quad 57$

3.1.6. Fatores que afetam a determinação do mecanismo de ruptura 57

3.1.7. Programa computacional - PLAXIS 2D 58

3.2. Influência da anisotropia na estabilidade de taludes 62

3.3. Dependência da resistência ao cisalhamento não drenado sobre a tensão $\begin{array}{ll}\text { principal intermediária } & 68\end{array}$

3.4. Resistência anisotrópica representativa para análises de estabilidade de taludes

4 Abordagem probabilística de estabilidade de taludes 73

4.1. Incertezas na engenharia geotécnica 73 
4.1.1. Variabilidade do solo

4.1.2. Tipos de incerteza

4.1.3. Variáveis aleatórias

4.2. Análises de confiabilidade

4.2.1. Margem de segurança e fator de segurança

4.2.2. Probabilidade de ruptura e o índice de confiabilidade

4.2.3. Probabilidade aceitável de ruptura na análise de confiabilidade

4.2.4. Probabilidade de ruptura considerando a distribuição normal

4.3. Modelos probabilísticos utilizados na análise de confiabilidade

4.3.1. Método de Monte Carlo

4.3.2. Método do Hipercubo Latino

4.3.3. Método das estimativas pontuais

4.4. Análise de sensibilidade

106

4.5. Diferenças entre as abordagens probabilísticas e determinísticas

110

5 Exemplos de aplicação

5.1. Exemplo $\mathrm{N}^{\circ} 1$ : Escavação aberta da rua "Congress"

5.1.1. Análise determinística

5.1.2. Análise probabilística

5.2. Exemplo $\mathrm{N}^{\circ} 2$ : Talude de solo homogêneo

126

5.2.1. Análise determinística

5.2.2. Análise probabilística

5.3. Exemplo $\mathrm{N}^{\circ} 3$ : Aterro de teste no deposito de "Champlain"

5.3.1. Análise determinística

5.3.2. Análise probabilística

5.4. Exemplo N4: Escavação em solo mole 147

$\begin{array}{ll}\text { 5.4.1. Análise determinística } & 148\end{array}$

5.4.2. Análise probabilística 153

5.5. Exemplo $\mathrm{N}^{\circ}$ 5: Construção incremental do aterro 157

5.5.1. Análise determinística 158

5.5.2. Análise probabilística 164

5.6. Exemplo $\mathrm{N}^{\circ}$ 6: Escavação a céu aberto na mina Robert. 166

5.6.1. Análise determinística 167 
6 Conclusões e Sugestões 177

6.1. Análise determinística 177

6.2. Análise probabilística 179

6.3. Conclusões gerais 180

6.4. Sugestões 181

Referências bibliográficas 182 


\section{Lista de figuras}

Figura 2.1 Pontos de mudança que definem a superfície de deslizamento (Adaptado de Celestino e Duncan, 1981).

Figura 2.20 esquema da aproximação analítica de Baker (Adaptado de Baker,1980)

Figura 2.3 Função densidade de probabilidade do fator de segurança e a probabilidade de ruptura. (Adaptado de Christian et al., 1994).

Figura 3.1 Curva típica de deslocamento nodal (adaptado de Donald e Giam, 1988)

Figura 3.2 Critério de ruptura do Mohr Coulomb com parâmetros de resistência efetiva e não drenados (adaptado de Bringreve et al., 2010)

Figura 3.3 A figura mostra a idéia principal de um modelo elástico perfeitamente plástico (adaptado de Bringreve et al., 2010)

Figura 3.4 Superfície de escoamento do modelo Cam Clay Modificado no plano p'-q (adaptado de Bringreve et al., 2010)

Figura 3.5 Anisotropia da resistência não drenada das argilas de Londres (adaptado de Lo, 1965)

Figura 3.6 Variação da resistência não drenada com a tensão de adensamento em ensaios do tipo HPS e VPS (adaptado de Duncan e Seed, 1966)

Figura 3.7 Sistema de tensões possíveis por meio de diversos equipamentos para ensaios $\mathrm{CK}_{0} \mathrm{U}$ (adaptado de Ladd, 1991 Depois de Germaine, 1982)

Figura 3.8 Variação da resistência não drenada no estado de deformação plana e compressão triaxial com a tensão de adensamento (adaptado de Duncan e Seed, 1966)

Figura 3.9 Relações da resistência não drenada normalizada para os principais ensaios de cisalhamento de laboratório (adaptado de Kulhawy e Mayne, 1990). 
Figura 3.10 Número de estabilidade vs. Ângulo do talude (adaptado de Lo, 1965).

Figura 4.1 Incertezas nas estimativas das propriedades do solo (Adaptado de Phoon e Kulhawy, 1999a).

Figura 4.2 Modelo de variabilidade espacial, aplicados a medidas de pressão de um dilatômetro (Adaptado de DeGroot,1996).

Figura 4.3 Variabilidade espacial inerente do solo, (Adaptado de Phoon e Kulhawy, 1999a).

Figura 4.4 Exemplo de distribuições de: (a) assimetria positiva e (b) assimetria negativa.

Figura 4.5 Função distribuição cumulativa CDF e função densidade de probabilidade FDP, de uma variável aleatória contínua.

Figura 4.6. a) Distribuição de capacidade $C$ e demanda D, ambos considerados como vaiáveis aleatórias. (b) Distribuição da margem de segurança $M S$ considerada como variável aleatória.

Figura 4.7 Probabilidade de ruptura $P_{f}$ versus 0 índice de confiabilidade $\beta$, para uma distribuição normal. (Adaptado de Christian et al.,1994)

Figura 4.8 Fluxograma do método de Monte Carlo (Adaptado de Wang et al., 2011).

Figura 4.9 Concentrações da função de densidade de probabilidade (adaptado de Rosenblueth, 1981).

Figura 4.10 Concentrações para uma função de duas variáveis aleatórias considerando $y_{1}[x]=0$ (adaptado de Rosenblueth, 1981).

Figura 4.11 Número de avaliações de FS, exigido por vários métodos probabilísticos (Adaptado de Fredlund e Fredlund, 2011).

Figura 4.12 Um modelos simples de análise de decisão para estabilidade de taludes (a) Diagrama de influência, (b) Árvore de decisão (Adaptado de Gitirana Jr., 2005).

Figura 4.13 Diagrama do evento probabilístico do tipo tornado para o modelo W-GHA (Adaptado de Fredlund e Fredlund, 2011).

Figura 5.1 Geometria do talude

Figura 5.2 Malha de elementos finitos 
Figura 5.3 Distribuição das tensões totais $\sigma_{\mathrm{x}}$.

Figura 5.4 Distribuição das tensões totais $\sigma_{y}$.

Figura 5.5 Distribuição das tensões cisalhantes $\sigma_{x y}$.

Figura 5.6 Distribuição das deformações cisalhantes $\gamma_{x y}$.

Figura 5.7 Malha deformada ampliada 4E-2 vezes.

Figura 5.8 Potenciais superfícies de ruptura determinadas pelo MEL e MEF.

Figura 5.9 Distribuição das deformações totais $\varepsilon_{x x}$.

Figura 5.10 Distribuição da probabilidade de ruptura pelo coeficiente de variação normalizado considerando uma distribuição de probabilidade Normal.

Figura 5.11 Variação do índice de confiabilidade pelo coeficiente de variação normalizado considerando uma distribuição de probabilidade Normal.

Figura 5.12 Relação entre o índice de confiabilidade e a probabilidade de ruptura para o Fator de segurança considerando uma distribuição de probabilidade normal.

Figura 5.13 Geometria do modelo

Figura 5.14 Malha de elementos finitos

Figura 5.15 Distribuição das tensões cisalhantes $\sigma_{x}$.

Figura 5.16 Distribuição das tensões cisalhantes $\sigma_{y}$.

Figura 5.17 Distribuição das tensões cisalhantes $\sigma_{x y}$.

Figura 5.18 Distribuição das deformações cisalhantes. 129

Figura 5.19 Vetores de deslocamento incremental

Figura 5.20 Potenciais superfícies de ruptura determinadas pelo MEL e MEF.

Figura 5.21 Distribuição das deformações totais $\varepsilon_{x x}$.

Figura 5.22 Porcentagem da área em extensão na superfície de ruptura em relação à inclinação do talude

Figura 5.23 Comparação dos métodos analisados entre a $P_{f}$ e o ângulo de inclinação do talude, considerando uma distribuição de probabilidade normal e log-normal.

Figura 5.24 Comparação dos métodos analisados entre a $\beta$ e o 
ângulo de inclinação do talude, considerando uma distribuição de probabilidade normal e log-normal.

Figura 5.25 Ensaios de laboratório que simulam as condições de tensão ao longo da superfície de ruptura (Adaptado de Bjerrum, 1973).

Figura 5.26 Geometria do modelo

Figura 5.27 Malha de elementos finitos

Figura 5.28 Distribuição das tensões totais $\sigma_{\mathrm{x}}$.

Figura 5.29 Distribuição das tensões totais $\sigma_{y}$.

Figura 5.30 Distribuição das tensões cisalhantes $\sigma_{x y}$.

Figura 5.31 Comparação entre o deslocamento vertical observado e o predito do aterro ( dados observados após La Rochelle et. al., 1974).

Figura 5.32 Comparação entre o deslocamento horizontal observado e o predito (dados observados após La Rochelle et. al., 1974).

Figura 5.33 Vetores do deslocamento incremental ampliado 3E2 vezes.

Figura 5.34 Malha deformada ampliada 2 vezes.

Figura 5.35 Distribuição das deformações totais principal $\varepsilon_{1}$.

Figura 5.37 Distribuição das deformações totais $\varepsilon_{\mathrm{xx}}$.

Figura 5.38 Otimização do número de iterações.

Figura 5.39 Distribuição da probabilidade de ruptura pelo coeficiente de variação normalizado considerando uma distribuição de probabilidade Normal.

Figura 5.40 Variação do índice de confiabilidade pelo coeficiente de variação normalizado considerando uma distribuição de probabilidade Normal.

Figura 5.41 Geometria do modelo

Figura 5.42 Malha de elementos finitos

Figura 5.43 Distribuição das tensões totais $\sigma_{x}$.

Figura 5.44 Distribuição das tensões totais $\sigma_{y}$

Figura 5.45 Distribuição das tensões cisalhantes $\sigma_{x y}$.

Figura 5.46 Distribuição das deformações totais principais $\varepsilon_{1}$. 
Figura 5.47 Vetores do deslocamento incremental.

Figura 5.48 Potenciais superfícies de ruptura determinadas pelo MEL e MEF.

Figura 5.49 Distribuição das deformações totais $\varepsilon_{x x}$.

Figura 5.50 Efeitos das etapas de escavação na zona de extensão.

Figura 5.51 Porcentagem da área em extensão na superfície de ruptura em relação á inclinação do talude

Figura 5.52 Análise de sensibilidade da análise determinística.

Figura 5.53 Análise de sensibilidade da análise probabilística.

Figura 5.54 Geometria do exemplo

Figura 5.55 Malha de elementos finitos

Figura 5.56 Distribuição das tensões totais $\sigma_{x}$.

Figura 5.57 Distribuição das tensões totais $\sigma_{\mathrm{y}}$.

Figura 5.58 Distribuição das tensões cisalhantes $\sigma_{x y}$.

Figura 5.59 Distribuição das deformações totais principais $\varepsilon_{1}$

Figura 5.60 Malha deformada ampliada 2E-3 vezes.

Figura 5.61 Potenciais superfícies de ruptura determinadas pelo MEL e MEF.

Figura 5.62 Comparação das superfícies de ruptura determinadas e observadas

Figura 5.63 Comparação entre os deslocamentos totais Uy, obtidos por fase única e análise incremental.

Figura 5.64 Comparação entre os deslocamentos totais Ux, obtidos por fase única e análise incremental.

Figura 5.65 Comparação entre as tensões totais $\sigma_{x x}$ e $\sigma_{y y}$, obtidos por fase única e análise incremental.

Figura 5,66 Distribuição das deformações totais $\varepsilon_{x x}$.

Figura 5.67 Análise de sensibilidade da análise determinística.

Figura 5.68 Análise de sensibilidade da análise probabilística

Figura 5.69 Geometria do modelo

Figura 5.70 Malha de elementos finitos 168

Figura 5.71 Distribuição das tensões totais $\sigma_{x}$.

Figura 5.72 Distribuição das tensões totais $\sigma_{y}$. 
Figura 5.73 Distribuição das tensões cisalhantes $\sigma_{x y}$.

Figura 5.74 Região plastificada do solo na sexta etapa de escavação.

Figura 5.75 Distribuição das deformações totais principais $\varepsilon_{1}$

Figura 5.76 Distribuição das deformações cisalhantes $\gamma_{x y}$

Figura 5.77 Potenciais superfícies de ruptura determinadas pelo MEL e MEF

Figura 5.78 Malhas de elementos finitos considerando distintas quantidades de elementos: (a) FSM-SRM1=802; (b) FSMSRM2=880; (c) FSM-SRM1=1249; (d) FSM-SRM1=2225).

Figura 5.79 Variação do FS considerando: LEM, MEF e as variações na quantidade de elementos que conformam a malha de elementos finitos do exemplo.

Figura 5.80 Influência do coeficiente de repouso $K_{0}$ no cálculo do $F S$.

Figura 5.81 Distribuição das deformações totais $\varepsilon_{x x}$

Figura 5.82 Valores de probabilidade de ruptura em função do coeficiente de correlação.

Figura 5.83 Resultado da análise de sensibilidade em uma direção considerando os parâmetros da coesão e o ângulo de atrito do solo.

Figura 5.84 Resultado da análise de sensibilidade em duas direções considerando os parâmetros da coesão e o ângulo de atrito do solo. 


\section{Lista de tabelas}

Tabela 2.1 Classificação dos métodos disponíveis na análise de estabilidade

Tabela 2.2 Suposições, condições de equilíbrio e incógnitas em procedimentos de Equilíbrio Limite (adaptado de Duncan e Wright, 2005)

Tabela 2.3 Influência da anisotropia para os casos: (a) solo homogêneo, (b) coesão aumentando linearmente com a profundidade (após Lo, 1965; Chen et al.,1975).

Tabela 3.1 Classificação do MEF para análises de estabilidade de taludes

Tabela 3.2 Parâmetro de tensão principal intermediária (b) e o ângulo de Lode.

Tabela 4.1 Fontes principais de incertezas na caracterização do solo

Tabela 4.2 Valores típicos para o coeficiente de variação (Adaptado de Lacasse e Nadim, 1996).

Tabela 4.3 Valores típicos para o coeficiente de variação (Adaptado de Lacasse e Nadim, 1996)

Tabela 4.4 Resumo da escala de flutuações de algumas propriedades geotécnicas (Adaptado de Phoon e Kulhawy, 1999a).

Tabela 4.5 Tipos de incertezas na caracterização do solo

Tabela 4.6 Probabilidade de ruptura aceitável na análise de estabilidade de taludes (Adaptado de Santamarina et al., 1992)

Tabela 5.1 Parâmetros geotécnicos gerais do material

Tabela 5.2 Fator de segurança determinado pelo MEL e MEF

Tabela 5.3 Parâmetros estatísticos de resistência não drenada.

Tabela 5.4 Resultados das análises determinísticas.

Tabela 5.5 Resultados das análises probabilísticas.

Tabela 5.6 Resultados da análise de sensibilidade pelo método 
de Monte Carlo.

Tabela 5.7 Resultados da análise de sensibilidade pelo método do Hipercubo Latino

Tabela 5.8 Resultados da análise de sensibilidade pelo MEPA

Tabela 5.9 Parâmetros geotécnicos do material

Tabela 5.10 Fator de segurança determinado pelo MEL e MEF

Tabela 5.11 Porcentagem da área em extensão em relação à inclinação do talude

Tabela 5.12 Parâmetros estatísticos de resistência não drenada.

Tabela 5.13 Resultados da análise probabilística

Tabela 5.14 Parâmetros geotécnicos gerais do material de fundação para o modelo Cam Clay Modificado.

Tabela 5.15 Parâmetros geotécnicos gerais do material do aterro para o modelo Mohr - Coulomb.

Tabela 5.16 Parâmetros estatísticos considerados na análise.

Tabela 5.17 Resultados das análises probabilísticas

Tabela 5.18 Parâmetros estatísticos usados na análise de sensibilidade.

Tabela 5.19 Resultados da análise de sensibilidade pelo método de Monte Carlo.

Tabela 5.20 Resultados da análise de sensibilidade pelo método do Hipercubo Latino.

Tabela 5.21 Resultados da análise de sensibilidade pelo MEPA.

Tabela 5.22 Parâmetros geotécnicos gerais do material.

Tabela 5.23 Fator de segurança determinado pelo MEL e MEF.

Tabela 5.24 Porcentagem da área de extensão em relação à inclinação do talude.

Tabela 5.25 Resultados da análise de variação da inclinação do talude.

Tabela 5.26 Resultados das análises probabilísticas por cada método analisado. 
Tabela 5.27 Resultados do diagrama tornado determinístico.

Tabela 5.28 Resultados do diagrama tomado probabilístico.

Tabela 5.29 Parâmetros geotécnicos gerais do material.

Tabela 5.30 Fator de segurança para cada etapa de lançamento.

Tabela 5.31 Parâmetros estatísticos considerados na análise. 164

Tabela 5.32 Resultados das análises probabilísticas 165

Tabela 5.33 Resultados do diagrama tornado evento probabilístico.

Tabela 5.34 Parâmetros geotécnicos gerais do material 167

Tabela 5.35 Fator de segurança para cada etapa de escavação $\quad 170$

Tabela 5.36 Fator de segurança considerando diferentes valores para $K_{0}$.

Tabela 5.37 Valores de Cov adotados para diversos casos de estabilidade de taludes.

Tabela 5.38 Parâmetros estatísticos 174

Tabela 5.39 Resultados das análises probabilísticas. 175 


\section{Lista de símbolos}

\begin{tabular}{|c|c|}
\hline$\alpha$ & inclinação da superfície de deslizamento para a horizontal \\
\hline$\beta$ & índice de confiabilidade \\
\hline$b$ & parâmetro da tensão principal intermediária. \\
\hline$C$ & distribuição de capacidade \\
\hline$\hat{C}$ & valores estimados da capacidade \\
\hline $\bar{C}$ & valores médios da capacidade \\
\hline$c_{u k}$ & resistência ao cisalhamento não drenada na ruptura \\
\hline$c$ & coesão \\
\hline$c_{h}$ & coesão horizontal \\
\hline$c_{v}$ & coesão vertical \\
\hline$\rho_{X Y}$ & correlação de duas variáveis \\
\hline $\operatorname{Cov}[\cdot]$ & coeficiente de variação \\
\hline$D$ & distribuição de demanda \\
\hline$\hat{D}$ & valores estimados da demanda \\
\hline $\bar{D}$ & valores médios da demanda \\
\hline$d$ & desvio padrão normal correspondente ao nível de confiança \\
\hline$E[\cdot]$ & valor esperado \\
\hline$E$ & módulo de young \\
\hline$e$ & índice de vazios \\
\hline$e_{\text {init }}$ & índice de vazios iniciais \\
\hline$\varepsilon_{i}$ & componente residual da variável aleatória \\
\hline$\varepsilon$ & nível de confiança desejado (0-100\%) \\
\hline$f(x)$ & função distribuição de probabilidade \\
\hline$F(x)$ & função densidade de probabilidade \\
\hline$f$ & ângulo entre a tensão principal menor e o plano de ruptura \\
\hline$y(x)$ & função de coordenadas \\
\hline$\gamma[x]$ & assimetria da variável aleatória \\
\hline$i$ & ângulo de inclinação \\
\hline$K_{0}$ & coeficiente de repouso do solo \\
\hline$K_{0}^{N C}$ & coeficiente de repouso para o solo normalmente adensado \\
\hline$K_{0}^{O C}$ & coeficiente de repouso para o solo pré-adensado \\
\hline
\end{tabular}




\begin{tabular}{|c|c|c|}
\hline & $K$ & grau de anisotropia \\
\hline & $\kappa$ & índice de inchamento \\
\hline & $\lambda$ & índice de compressão \\
\hline & $M$ & tangente da linha de estado crítico \\
\hline & $\mu_{x}$ & média da variável aleatória \\
\hline & $m$ & número de variáveis aleatórias de entrada \\
\hline & $N_{s}$ & número de estabilidade \\
\hline & $N$ & número de tentativas requeridas pelo $\mathrm{MCM}$ \\
\hline & $N$ & fator de modificação da resistência \\
\hline & $v$ & coeficiente de poisson \\
\hline & $P_{f}$ & probabilidade de ruptura \\
\hline & $p^{\prime}$ & tensão média efetiva \\
\hline & $p_{c}$ & tensão de pré-adensamento \\
\hline$\frac{9}{4}$ & $\phi$ & ângulo de atrito \\
\hline$\underset{\substack{N \\
N}}{\stackrel{5}{2}}$ & $\phi_{h}$ & ângulo de atrito horizontal \\
\hline 음 & $\phi_{v}$ & ângulo de atrito vertical \\
\hline$\frac{\leq}{\underline{\underline{\pi}}}$ & $\psi$ & ângulo de dilatância \\
\hline 前 & $q$ & tensão de desvio \\
\hline 莺, & $\rho$ & coeficiente de correlação \\
\hline 衰 & $s_{h}$ & resistências ao cisalhamento nas direções horizontal \\
\hline $\begin{array}{l}0 \\
1 \\
0\end{array}$ & $s_{v}$ & resistências ao cisalhamento nas direções vertical \\
\hline 㐫 & $s$ & resistências ao cisalhamento \\
\hline & $s_{u}^{T C}$ & $\begin{array}{l}\text { resistência ao cisalhamento não drenada em compressão } \\
\text { triaxial }\end{array}$ \\
\hline & $s_{u}^{P S}$ & $\begin{array}{l}\text { resistência ao cisalhamento não drenada em deformação } \\
\text { plana }\end{array}$ \\
\hline & $\sigma$ & desvio padrão \\
\hline & $\sigma_{1}$ & tensão principal maior na direção vertical \\
\hline & $\sigma_{3}$ & tensão principal menor na direção horizontal \\
\hline & $t_{i}$ & tendência de uma variável aleatória \\
\hline & $\tau_{f}$ & tensão de cisalhamento \\
\hline & $\theta$ & ângulo da tensão principal maior com a horizontal \\
\hline & $b$ & ângulo de Lode \\
\hline & $\operatorname{Var}[\cdot]$ & variância \\
\hline & $X$ & variável aleatória \\
\hline & $x_{i}$ & variação espacial das propriedades do solo \\
\hline
\end{tabular}




\section{Lista de abreviaturas}

MEPA método das estimativas pontuais alternativas

MSB método de Bishop simplificado

CDF função densidade cumulativa

FEA análise dos elementos finitos

MEF método dos elementos finitos

FOSM método do segundo momento de primeira ordem

FS fator de segurança

GIM método do incremento da gravidade

HLM método do hipercubo latino

HPS compressão horizontal de deformação plana

LAM métodos da análise limite

LEM método de equilíbrio limite

MCM método de monte carlo

MDN método dos deslocamentos nodais

OCR razão de pré-adensamento

FDP função densidade de probabilidade

SM margem de segurança

SOF escala de flutuação

MRR método da redução da resistência

VPS compressão vertical de deformação plana 